Gazi University
Journal of Science
http://dergipark.gov.tr/gujs

\title{
Comparative Analysis of Optical Multicarrier Modulations: An Insight into Machine Learning-based Multicarrier Modulation
}

\author{
Augustus E. IBHAZE ${ }^{1 *}$, Frederick O. EDEKO ${ }^{2}$, , Patience E. ORUKPE ${ }^{2}$ (D) \\ ${ }^{1}$ University of Lagos, Department of Electrical and Electronics Engineering, Akoka, Lagos, Nigeria \\ ${ }^{2}$ University of Benin, Department of Electrical and Electronics Engineering, Benin City, Nigeria \\ Highlights \\ - This paper focuses on the comparative study of optical multicarrier modulation schemes. \\ - A machine learning-based multicarrier modulation is proposed for optical signal conditioning. \\ - An optimal system performance with reduced bit error rate response was achieved.
}

\begin{tabular}{l} 
Article Info \\
\hline Received:27 July 2020 \\
Accepted:27 Jan 2021 \\
Keywords \\
\hline Bit error rate \\
Machine learning \\
Multicarrier modulation \\
Signal to noise ratio
\end{tabular}

\section{INTRODUCTION}

Multicarrier technique has been well validated for application at radio frequency spectrum as a physical layer standard for improved data rates up to megabits per second, although marked with some limitations in its spectral/power efficiency, signal to noise ratio/bit error rate (SNR/BER) and limited bandwidth requirement [1-3]. Following directly from this assertion is the larger bandwidth potential of the visible light spectrum which makes it attractive for data rates up to gigabits per second and beyond [4]. The keen interest in adapting radio frequency (RF)-based multicarrier scheme to visible light based multicarrier scheme does not come by easily due to the inherent characteristics of the signal conditioning requirements in the optical communication domain. For RF application, the signal is complex (with real and imaginary signal components) and bipolar (positive and negative signal cycle) and must be transmitted via electrical fields while being coherently detected. Conversely, for visible light application, the signal is real and unipolar (positive) and can only be transmitted via optical intensity and received through direct detection [5]. This has left the adoption of multicarrier modulation with orthogonal frequency division multiplexing (OFDM) to ingenious investigations and implementation in the visible light communication plane. Since the bandwidth requirement of the radio frequency spectrum places a natural limit on the achievable throughput following directly from Shannon's investigation on error free transmission [6], the enormously large bandwidth of the visible light spectrum stands as an immediate and complementary solution to the spectral crunch of radio frequency spectrum [7].

The design approach to improving throughput follows directly from the exploitation of the incoherent nature of the optical signal in contrast with coherent radio frequency signal. In radio frequency domain, 
signal transmission is established through the electric field by varying the carrier's amplitude, frequency and phase. In a similar vein, for signal recovery, the carrier phase information of the transmitted signal will be required by the receiver for detection and error free signal reconstruction. This coherence requirement of the radio frequency system results in RF receiver design complexity since the phase difference has to be analyzed and corrected. In this work, the carrier phase information will not be required by the receiver since the transmitting source emits optical energy with changing phase. The receiver design complexity will be greatly reduced since optical intensity can be directly detected using a photodetector. The implication of this non-coherent signal reception is that the signal must be real and unipolar based on optical intensity, unlike the radio frequency signal which is complex and bipolar based on the component property of signal variation in the electric field. The conventional communication system comprises of three basic component parts; the transmission, channel and reception module [8]. The design of these schemes depends largely on user experience, expectations and local conditions. As users become more data hungry, the need to rollout "more-broadband" technologies will become inevitable. And as such, existing techniques will have to evolve into newer standards to cater for the increasing demand which comes with newer design challenges. In terms of local conditions, the channel being line-of-sight (LOS) since signals will have to be directly detected, channel parameters and propagation conditions also impose some constraints which necessitates some level of ingenuity in signal processing and system design for error-free transmission.

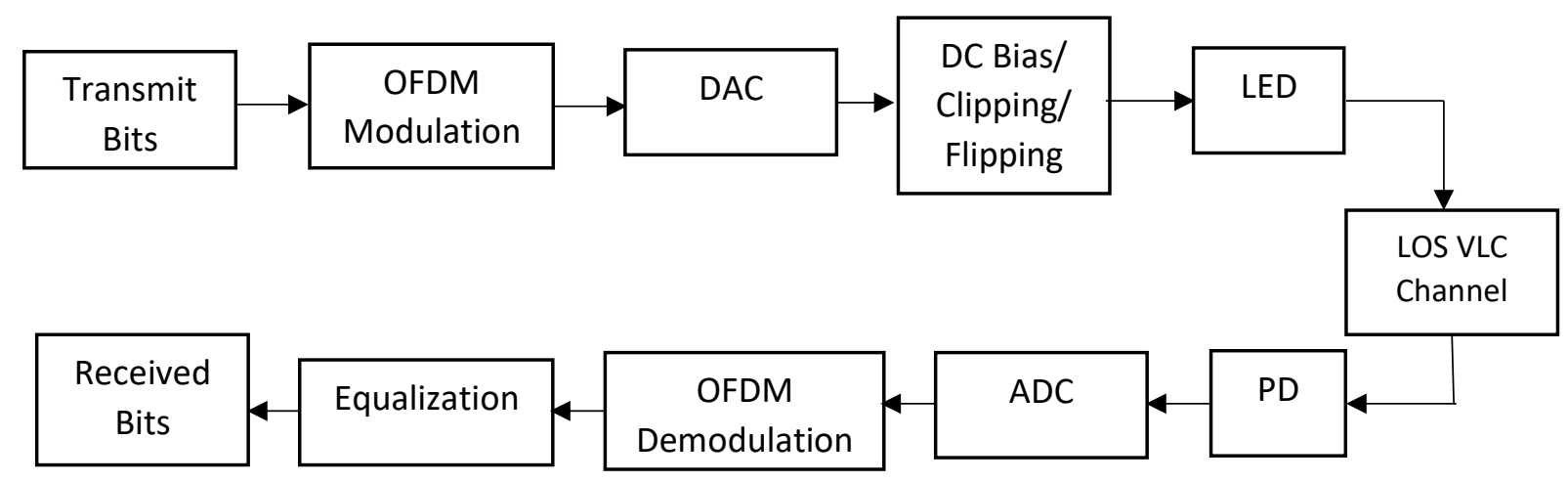

Figure 1. Conventional Multicarrier Scheme for VLC [9]

The fundamental difference between the conventional scheme shown in Figure 1 and its radio frequency counterpart is the process of generation of the real and unipolar signal required to drive the light emitting diode (LED) transmitter and the direct detection module. The limitations imposed by the performancedegrading effect of the high peak power associated with the OFDM scheme is also inherited by this model. To improve upon the system performance, the communication link will require further processing scheme to minimize the level of degradation while improving the system throughput.

\section{MULTICARRIER MODELING FOR VISIBLE LIGHT COMMUNICATION (VLC)}

Multicarrier techniques applies the strategy of splitting a high rate bit stream into a finite number of low rate bit stream that can be transmitted over a finite number of subcarriers [10]. The bit stream is first transmitted through a serial-to-parallel converter so that the encoded parallel bit stream can be modulated on to the subcarriers using Inverse Fast Fourier Transform (IFFT). The inclusion of guard intervals and cyclic prefix helps in the elimination of inter-symbol interference and inter-carrier interference respectively [11]. At the receiving end, frequency offset estimation and symbol timing is done so that Fast Fourier Transform (FFT) is applied on the symbols to detect the modulated subcarriers. A typical multicarrier technique used in wireless communication is orthogonal frequency division multiplexing (OFDM). In VLC variant of OFDM technique is being researched as possible technique for high throughput transmission.

Assuming that a single OFDM block is allocated a total number of $\mathrm{N}$ subcarriers, the serial bit-stream from the transmitter is first converted to parallel bit-streams that can be modulated separately by the subcarriers, mapped to the complex valued symbol (N/2)-1, in relation to the modulation constellation of the selected 
scheme. The modulated OFDM block in relation to the subcarrier's index is therefore given by:

$X=\left[X_{l}\right] \quad \forall \quad l=0,1,2, \ldots, N-1$.

where the subscript $l=0,1,2, \ldots, N-1$ represent the subcarrier index in relation to the allocated subcarriers.

To model a multicarrier signal conditioning scheme operating at a frequency $f_{o}$, it is to be noted that the scheme denotes multiple carriers such that the cumulative effect is the sum total of the distinctive subcarriers given as;

$$
x_{t}=\sum_{l=0}^{N-1} X_{l} e^{\left(j 2 \pi l f_{o} t\right)} \quad \forall l=0,1,2, \ldots, N-1 .
$$

where $X_{l}$ is the $l^{\text {th }}$ subcarrier transmitted symbol, $l f_{o} t$ is the frequency component of the $l^{\text {th }}$ subcarrier and $l$ denotes the subcarrier index.

Since the given spectrum or bandwidth $B$ is bandlimited, it can be sampled at Nyquist rate:

$2 f_{\max }=2 \cdot \frac{B}{2}=B$

The sampling duration or interval is given as the reciprocal of the sampling frequency $\frac{1}{B}$, such that the $m^{t h}$ sampling instant becomes:

$m \cdot \frac{1}{B}=\frac{m}{B}$

Since the total number of subcarriers for the multicarrier system is the same as $N$, then, the given bandwidth will be shared by all $N$ subcarriers, so that the bandwidth of each subcarrier becomes:

$f_{o}=\frac{B}{N}$

Hence, Equation (2) on applying Equations (4) and (5) become:

$x_{m}=\sum_{l=0}^{N-1} X_{k} e^{\left(j 2 \pi k \frac{B}{N} \cdot \frac{m}{B}\right)}$

$x_{m}=\sum_{l=0}^{N-1} X_{l} e^{\left(\frac{2 \pi l m}{N}\right)} \quad \forall \quad l=0,1,2, \ldots, N-1$.

Equation (7) is the multicarrier symbol. When impressed on the IFFT processor, the eventual output being discrete time domain samples will yield the OFDM symbol of the $l^{\text {th }}$ subcarrier expressed as:

$x_{m}=\frac{1}{\sqrt{N}} \sum_{l=0}^{N-1} X_{l} e^{\left(j \frac{2 \pi l m}{N}\right)} \quad \forall m, l=0,1,2, \ldots, N-1$

where $x_{m}$, is the $m^{t h}$ time domain discrete sample and $\frac{1}{\sqrt{N}}$ is a scale factor [12]. But for visible light applications with intensity modulation and direct detection, the OFDM signal must be represented by light intensity. The implication of this property demands the modulating signal be real and unipolar (positive) as opposed to the complex bipolar representation of the OFDM signal given by Equation (8). Since the total number of subcarriers used is the same as $N / 2-1, x_{m}$ is derived as:

$x_{m}=\frac{1}{\sqrt{N}} \sum_{l=0}^{N / 2-1}\left(X_{l} e^{\left(j \frac{2 \pi l m}{N}\right)}+X_{N-l} e^{\left(j \frac{2 \pi(N-l) m}{N}\right)}\right)$. 
In order to suppress the imaginary component of the IFFT processor output, Hermitian symmetry is imposed. Consequently, to achieve real-valued time domain signal from the complex valued OFDM signal, the allocated subcarriers are designed such that the subcarriers with subscript $l=N / 2$ to $N-1\left(X_{N / 2}\right.$ to $X_{N-1}$ ) are constrained to satisfy Hermitian symmetry such that:

$X_{l}=X_{N-l}^{*} \quad \forall l=N / 2, \ldots, N-1$

where the superscript “*” connotes the complex conjugate of the specified subcarrier. Hence,

$x_{m}=\frac{1}{\sqrt{N}} \sum_{l=0}^{N / 2-1}\left(X_{l} e^{\left(j \frac{2 \pi l m}{N}\right)}+X_{l}^{*} e^{\left(-j \frac{2 \pi(N-l) m}{N}\right)}\right)$.

The real component therefore derives directly from Equation (11) since the imaginary component is being suppressed or forced to zero. Hence:

$x_{m}=\frac{2}{\sqrt{N}} \sum_{l=0}^{N / 2-1} \operatorname{Re}\left(X_{l} e^{\left(j \frac{2 \pi l m}{N}\right)}\right) \quad \forall m, l=0,1,2, \ldots, N-1$.

The basic property that describes the superiority of multicarrier scheme over single carrier technique is its robustness in mitigating ISI effect over a dispersive channel by the simple integration of cyclic prefix. After IFFT operation, cyclic prefix of a specified length $L_{c y p}$ is added in front of the OFDM block which must exceed the maximum delay spread of the dispersive channel to effectively eliminate ISI effect. The time domain discrete samples $x_{m}$ are then serialized by converting them to electrical signal $x_{t}$ using a digitalto-analog converter (DAC). It is to be noted that the eventual output at this stage is still bipolar and infeasible for intensity modulation. The next phase of adapting the bipolar OFDM signal is to achieve a unipolar signal of the real valued version of the OFDM samples. In order to eliminate the undesirable effect of direct current $(\mathrm{dc})$ and complex valued harmonics, the modulated OFDM block is constructed such that the subcarriers with subscript $k=0$ and $k=N / 2$ are set to zero, that is:

$X_{0}=X_{N / 2}=0$

The remaining subcarriers $X_{1}$ to $X_{N / 2-1}$, are used to convey the information symbols.

\subsection{Direct Current-biased Optical (DCO) OFDM}

To derive the unipolar version of the bipolar OFDM signal $x_{t}$, a dc-bias $\mathcal{B}_{d c}$ is added to the signal to force the signal to be non-negative, derived in relation to the standard deviation of $x_{t}$ [13], such that:

$\mathcal{B}_{d c} \propto \sigma_{d}$

$\sigma_{d}=\sqrt{E\left(x_{t}^{2}\right)}$

$\mathcal{B}_{d c}=\mu \sigma_{d}=\mu \sqrt{E\left(x_{t}^{2}\right)}$

where $\mu$ is a constant of proportionality and $\sigma_{d}$ is the standard deviation being the expectation $E($.$) of the$ OFDM signal. The level of bias $\mathcal{B}_{d c}$ is estimated as:

$\beta=10 \log \left(\mu^{2}+1\right) d B$.

Since the spectral plane is shifted by dc-bias, the negative amplitudes of the biased signals are therefore clipped to zero which further results in clipping distortion or noise $n_{c}$. The ensuing unipolar electrical DCO signal used to drive the LED for intensity modulation becomes: 
$x_{D C O}=x_{t}+\mathcal{B}_{d c}+n_{c}$.

In order to raise the negative peaks to zero, high dc biasing level will be required which makes this scheme power inefficient although spectrally efficient since the entire subcarriers can be used as symbol carriers. At $N \geq 64$, that is; for sufficiently large number of subcarriers, the signal $x_{t}$ approximately models a zero mean Gaussian distribution based on the central limit theorem [14], having a variance of:

$\sigma_{d}^{2}=E\left(x_{t}^{2}\right)$

where, $\sigma_{d}$ is the standard deviation of the signal $x_{t}$.

Since the power density function (pdf) models a Gaussian distribution given by [15]:

$f_{X, D C O}(\vartheta)=\frac{1}{\sqrt{2 \pi} \sigma_{D C O}} \exp \left(-\frac{\left(\vartheta-\mathcal{B}_{d c}\right)^{2}}{2 \sigma_{D C O}^{2}}\right) u(\vartheta)+Q\left(\frac{\mathcal{B}_{d c}}{\sigma_{D C O}}\right) \delta(\vartheta)$

where $u(\vartheta)$ and $\delta(\vartheta)$ are unit step and Dirac delta functions respectively. The optical power of the DCO scheme $P_{O, D C O}$ follows directly from the pdf as:

$$
\begin{aligned}
& P_{o, D C O}=E\left(x_{t}\right)=\int_{0}^{\infty} \vartheta f_{X, D C O}(\vartheta) d \vartheta \\
& P_{o, D C O}=\int_{0}^{\infty} \vartheta\left(\frac{1}{\sqrt{2 \pi} \sigma_{D C O}} \exp \left(-\frac{\left(\vartheta-\mathcal{B}_{d c}\right)^{2}}{2 \sigma_{D C O}^{2}}\right) u(\vartheta)+Q\left(\frac{\mathcal{B}_{d c}}{\sigma_{D C O}}\right) \delta(\vartheta)\right) d \vartheta
\end{aligned}
$$

Given that $\mathrm{Q}-$ function $\mathrm{Q}(\mathrm{x})$ is given by

$Q(x)=\frac{1}{\sqrt{2 \pi}} \int_{x}^{\infty} \exp \left(-\frac{x^{2}}{2}\right) d x$

Then, the optical DCO scheme power will become:

$$
P_{o, D C O}=\frac{\sigma_{D C O}}{2 \pi} \exp \left(\frac{-\mathcal{B}_{d c}^{2}}{2 \sigma_{D C O}^{2}}\right)+\mathcal{B}_{d c}\left(1-Q\left(\frac{\mathcal{B}_{d c}}{\sigma_{D C O}}\right)\right) \text {. }
$$

The electrical power $P_{e, D C O}$ of the DCO scheme is given by [16]:

$$
\begin{aligned}
& P_{e, D C O}=E\left(x_{t}^{2}\right)=\int_{0}^{\infty} \vartheta^{2} f_{X D C O}(\vartheta) d \vartheta \\
& P_{e, D C O}=\int_{0}^{\infty} \vartheta^{2}\left(\frac{1}{\sqrt{2 \pi} \sigma_{D C O}} \exp \left(-\frac{\left(\vartheta-\mathcal{B}_{d c}\right)^{2}}{2 \sigma_{D C O}^{2}}\right) u(\vartheta)+Q\left(\frac{\mathcal{B}_{d c}}{\sigma_{D C O}}\right) \delta(\vartheta)\right) d \vartheta \\
& P_{e, D C O}=\frac{\sigma_{D C O} \mathcal{B}_{d c}}{\sqrt{2 \pi}} \exp \left(\frac{-\mathcal{B}_{d c}^{2}}{2 \sigma_{D C O}^{2}}\right)+\left(\mathcal{B}_{d c}^{2}+\sigma_{D C O}^{2}\right)\left(1-Q\left(\frac{\mathcal{B}_{d c}}{\sigma_{D C O}}\right)\right)
\end{aligned}
$$

At the receiving end, the photo-detector converts the received optical signal into electrical signal $y_{t}$ given as:

$y_{t}=h_{t} * x_{D C O}+n_{w}$

where $h_{t}$ is the channel impulse response, $*$ denotes convolution and $n_{w}$ models the additive white Gaussian noise with zero mean. The received signal $y_{t}$ is then fed into the analog-to-digital converter (ADC) to obtain the discrete equivalent of the signal $y_{m}$ which is then de-cyclic prefixed and parallelized. That is:

$y_{m} ; \forall m=0,1,2,3, \ldots, N-1$. 
An N-point FFT is used to transform the time domain samples into frequency domain samples to enable the recovery of the transmitted symbols. That is:

$Y_{l} ; \forall l=0,1,2,3, \ldots, N-1$.

Since the subcarriers indicated by $l=0,1,2,3, \ldots, N-1$ are narrowband channels, they experience flat fading such that single tap equalization technique becomes sufficient for estimation. Since subcarriers $l=$ $0,1,2,3, \ldots, N / 2-1$ are used for information symbol transmission, the equalizer divides each subcarrier received symbol by its associated channel state information (CSI) for signal recovery. In a similar vein, the adoption of machine learning for channel estimation as proposed in this work can greatly improve the BER response and ultimately the optimal state of the recovered signal by training the distorted received signal using ANN. The dataset training will help in the correlation of the distorted output and the transmitted signal in relation to the predefined BER threshold to minimize the bit error rate.

The signal to noise ratio for the DCO scheme in relation to the optical $S N R_{o}$ and electrical power $S N R_{e}$ are given respectively by:

$S N R_{o, D C O}=\frac{E\left(x_{t}\right)}{b_{D C O} N_{o}}$

and

$S N R_{e, D C O}=\frac{E\left(x_{t}^{2}\right)}{b_{D C O} N_{o}}$

where $E\left(x_{t}\right)$ is the optical power, $E\left(x_{t}^{2}\right)$ is the electrical power, $b_{D C O}$ is the throughput of the DCO scheme and $N_{o}$ is the noise power. The bit error rate can therefore be estimated in relation to the signal to noise ratio for the DCO scheme.

By way of extension, the average bit error rate, $B E R_{\text {ave }}$ is defined as the ratio of the transmitted bit in error to the total bits transmitted [17]. Such that:

$B E R_{\text {ave }}=\frac{\sum_{l=1}^{N / 2-1} b_{l} B E R_{l}}{\sum_{l=1}^{N / 2-1} b_{l}}$

where $b_{l}$ is the transmitted bits per subcarrier, $B E R_{l}$, is the bit error rate per subcarrier and $l$ is the subcarrier index.

Hence, in terms of DCO scheme, average BER becomes:

$B E R_{\text {ave }}=\frac{\sum_{l=1}^{N / 2-1} b_{l, D C O} B E R_{l}}{\sum_{l=1}^{N / 2-1} b_{l, D C O}}$.

Hence, from Equation (34), the total DCO scheme transmits bit is given by:

$b_{D C O}=\sum_{l=1}^{N / 2-1} b_{l, D C O}=\frac{\sum_{l=1}^{N / 2-1} b_{l, D C O} B E R_{l}}{B E R_{\text {ave }}}$.

By combining Equations (31), (32) and (35), the relationship between the SNR and BER is derived as:

$$
\begin{aligned}
& S N R_{O, D C O}=\frac{E\left(x_{t}\right)}{\frac{\sum_{l=1}^{N / 2-1} b_{l, D C O} B E R_{l}}{B E R_{\text {ave }}} N_{O}} \\
& S N R_{O, D C O}=\frac{B E R_{\text {ave }} \cdot E\left(x_{t}\right)}{N_{o} \sum_{l=1}^{N / 2-1} b_{l, D C O} B E R_{l}}
\end{aligned}
$$




$$
\begin{aligned}
& S N R_{e, D C O}=\frac{E\left(x_{t}^{2}\right)}{\frac{\sum_{l=1}^{N / 2-1} b_{l, D C O} B E R_{l}}{B E R_{a v e}} N_{o}} \\
& S N R_{e, D C O}=\frac{B E R_{\text {ave }} \cdot E\left(x_{t}^{2}\right)}{N_{o} \sum_{l=1}^{N / 2-1} b_{l, D C O} B E R_{l}} .
\end{aligned}
$$

\subsection{Asymmetrically Clipped Optical (ACO) OFDM}

To improve on power efficiency, ACO-OFDM was modeled as a modification of the optical multicarrier signal conditioning scheme. This model was able to achieve improved power efficiency by exploiting the odd subcarriers while sacrificing the even subcarriers to cater for clipping distortion as the means to achieving the unipolar signal. Since dc-bias is not required, there is a considerable tradeoff between power and spectral efficiency as the spectral efficiency is considerably reduced due to the clipping of even subcarriers. Hence only half of the total subcarriers required for symbol transmission are used. That is, onehalf of $N / 2$ being $N / 4$ subcarriers can be used for symbol transmission for an OFDM block of $N$ subcarrier. Since only odd subcarriers are used, $X_{l}$ of Equation (8) will become:

$X_{l}=X_{2 n+1} \quad \forall n=0,1,2,3, \ldots, N / 2-1$

so that the frequency domain signal becomes:

$X=\left[0, X_{1}, 0, X_{3}, 0, X_{5}, \ldots, X_{N / 2-1}, 0, X_{N / 2-1}^{*}, \ldots, X_{5}^{*}, 0, X_{3}^{*}, 0, X_{1}^{*}\right]$.

Hence for ACO-OFDM scheme in terms of $N / 2-1$ subcarriers used for symbol transmission, Equation (8) becomes:

$x_{m}=\frac{1}{\sqrt{N}} \sum_{l=0}^{N / 2-1} X_{2 n+1} e^{\left(j \frac{2 \pi(2 n+1) m}{N}\right)} \quad \forall m=0,1,2, \ldots, N-1$

where $X_{2 n+1}$, denotes the $(2 n+1)^{t h}$ odd component of $X$. By the antisymmetric property of $x_{m}$ as postulated by [14], the amplitude samples of the two-halves on the spectral plane are identical with opposite sign convention such that Equation (42) is the same as:

$x_{m}=-\frac{1}{\sqrt{N}} \sum_{l=0}^{N / 2-1} X_{2 n+1} e^{\left(j \frac{2 \pi(2 n+1) m}{N}\right)} \forall m=0,1,2, \ldots, N-1$.

Since the symbols are only placed on the odd subcarriers up to $N / 2$ subcarriers, Equation (43) becomes:

$x_{m}=-\frac{1}{\sqrt{N}} \sum_{l=0}^{N / 2-1} X_{2 n+1} e^{\left(j \frac{2 \pi(2 n+1)(m+N / 2)}{N}\right)} \quad \forall m=0,1,2, \ldots, N-1$.

Hence, for $m+N / 2$, Equation (44) therefore implies that:

$x_{m}=-x_{m+N / 2} \quad \forall m=0,1,2,3, \ldots, N / 2-1$.

The implication is that the two halves of the amplitude samples carry the same information. Therefore, onehalf of the data samples can be clipped to zero without necessarily sacrificing the transmitted data. If the clipped sample is denoted by $x_{n, c}$, then:

$x_{n, c}=\left\{\begin{array}{ll}0 & \forall x_{n} \leq 0 \\ x_{n} & \forall x_{n} \geq 0\end{array}\right.$. 
At the receiving end, the received data symbols on the odd subcarriers will be given as:

$X_{2 n+1, c}=\frac{1}{\sqrt{N}} \sum_{l=0}^{N / 2-1} x_{n, c} e^{\left(-j \frac{2 \pi(2 n+1) m}{N}\right)}$.

For symbol distribution on odd subcarriers up to $N / 2$, we have:

$X_{2 n+1, c}=\frac{1}{\sqrt{N}} \sum_{l=0}^{N / 2-1}\left(x_{n, c}-x_{n, c+N / 2}\right) e^{\left(-j \frac{2 \pi(2 n+1) m}{N}\right)}$.

On clipping one-half of the total available subcarriers for symbol transmission, Equation (48) reduces by half to obtain:

$X_{2 n+1, c}=\frac{1}{2 \sqrt{N}} \sum_{l=0}^{N / 2-1}\left(x_{n}-x_{n+N / 2}\right) e^{\left(-j \frac{2 \pi(2 n+1) m}{N}\right)}$.

By the sign convention of Equation (45), Equation (49) becomes:

$$
\begin{aligned}
& X_{2 n+1, c}=\left(\frac{1}{2 \sqrt{N}} \sum_{l=0}^{N / 2-1} x_{n} e^{\left(-j \frac{2 \pi(2 n+1) m}{N}\right)}\right)-\left(-\frac{1}{2 \sqrt{N}} \sum_{l=0}^{N / 2-1} x_{n+N / 2} e^{\left(-j \frac{2 \pi(2 n+1)(m+N / 2)}{N}\right)}\right) \\
& X_{2 n+1, c}=\left(\frac{1}{2 \sqrt{N}} \sum_{l=0}^{N / 2-1} x_{n} e^{\left(-j \frac{2 \pi(2 n+1) m}{N}\right)}\right)+\left(\frac{1}{2 \sqrt{N}} \sum_{l=0}^{N / 2-1} x_{n+N / 2} e^{\left(-j \frac{2 \pi(2 n+1)(m+N / 2)}{N}\right)}\right) .
\end{aligned}
$$

Hence, the recovered distorted data symbol is one-half the transmitted data symbol and is given as:

$X_{2 n+1, c}=\frac{1}{2} X_{2 n+1}$

In relation to the central limit theorem, when the total number of subcarriers is sufficiently large, $(N \geq 64)$, the transmitted time-domain samples $x_{m}$, will approximate a Gaussian distribution [14], so that the pdf of the distribution of the ACO-OFDM scheme may be written as:

$f_{X, A C O}\left(x_{m, c}\right)= \begin{cases}\frac{1}{2} & \forall x_{m, c}=0 \\ \frac{1}{\sqrt{2 \pi} \sigma_{A C O}} \exp \left(-\frac{\left(x_{m, c}\right)^{2}}{2 \sigma_{A C O}^{2}}\right) & \forall x_{m, c}>0\end{cases}$

which can be rewritten as:

$f_{X, A C O}\left(x_{m, c}\right)=\frac{1}{\sqrt{2 \pi} \sigma_{A C O}} \exp \left(-\frac{\left(x_{m, c}\right)^{2}}{2 \sigma_{A C O}^{2}}\right) u\left(x_{m, c}\right)+\frac{1}{2} \delta\left(x_{m, c}\right)$

where all the parameters have their usual meanings.

The optical and electrical powers of the ACO scheme are given by [15]:

$P_{o, A C O}=E\left(x_{m, c}\right)=\int_{0}^{\infty} x_{m, c} f_{X, A C O}\left(x_{m, c}\right) d x_{m, c}=\frac{\sigma_{A C O}}{\sqrt{2 \pi}}$

and

$P_{e, A C O}=E\left(x_{m, c}^{2}\right)=\int_{0}^{\infty} x_{m, c}^{2} f_{X, A C O}\left(x_{m, c}\right) x_{m, c}=\frac{\sigma_{A C O}^{2}}{2}$.

At the receiving end of the ACO-OFDM scheme, only the odd symbols are fed to the equalizer/demapper after FFT operation. The eventual optical and electrical SNR of the ACO-OFDM scheme are given respectively as: 
$S N R_{O, A C O}=\frac{E\left(x_{m, c}\right)}{b_{A C O} N_{o}}$.

and

$S N R_{e, A C O}=\frac{E\left(x_{m, c}^{2}\right)}{b_{A C O} N_{o}}$.

where, $b_{A C O}$ is the throughput of the ACO-OFDM scheme while all other parameters assume their usual meaning.

The relationship between average BER and the SNR for the ACO scheme follows directly from the derivations in Equation (36) to Equation (39) and are given as follows

$$
\begin{aligned}
& S N R_{o, A C O}=\frac{E\left(x_{m, c}\right)}{\frac{\sum_{l=1}^{N / 2-1} b_{l, A C O} B E R_{l}}{B E R_{a v e}} N_{o}} \\
& S N R_{o, A C O}=\frac{B E R_{\text {ave }} \cdot E\left(x_{m, c}\right)}{N_{o} \sum_{l=1}^{N / 2-1} b_{l, A C O} B E R_{l}} \\
& S N R_{e, A C O}=\frac{E\left(x_{m, c}^{2}\right)}{\frac{\sum_{l=1}^{N / 2-1} b_{l, A C O} B E R_{l}}{B E R_{\text {ave }}} N_{O}} \\
& S N R_{e, D C O}=\frac{B E R_{\text {ave }} \cdot E\left(x_{m, c}^{2}\right)}{N_{o} \sum_{l=1}^{N / 2-1} b_{l, A C O} B E R_{l}} .
\end{aligned}
$$

\subsection{Flip-OFDM}

Flip OFDM scheme was derived to improve on both power and spectral efficiency of the communication system by avoiding the clipping of one-half of the subcarriers and dc-biasing in relation to ACO and DCO schemes respectively [18-19]. In this case, polarity separator is used to decompose the real bipolar signal after IFFT operation into two separate components of opposing polarities, separately transmitted on two different OFDM symbols to achieve the unipolar signal. In this case, the positive signal $x_{m}^{+}$is transmitted on the first sub-frame while the flipped signal $x_{m}^{-}$is transmitted on the second sub-frame thereby doubling the OFDM block length $(2 N)$ unlike for DCO and ACO being $N$ block length. The decomposed signals then become:

$x_{m}=x_{m}^{+}+x_{m}^{-}$

such that;

$x_{m}^{+}=x_{0,}^{+}, x_{1}^{+}, x_{2}^{+}, \ldots, x_{N-1}^{+} \quad \forall 0 \leq m \leq N-1$

and

$x_{m}^{-}=x_{0,}^{-}, x_{1}^{-}, x_{2}^{-}, \ldots, x_{N-1}^{-} \quad \forall 0 \leq m \leq N-1$.

The positive $x_{m}^{+}$and negative $x_{m}^{-}$signal components can then be defined as:

$x_{m}^{+}=\left\{\begin{array}{lll}x_{m} & \forall & x_{m} \geq 0 \\ 0 & \forall & x_{m}<0\end{array}\right.$ 
$x_{m}^{-}=\left\{\begin{array}{lll}x_{m} & \forall & x_{m}<0 \\ 0 & \forall & x_{m} \geq 0\end{array}\right.$.

Hence, the optical and electrical power of FLIP scheme is given by [20].

$P_{o, F L I P}=E\left(x_{m}^{+}\right)=E\left(x_{m}^{-}\right)=\kappa \int_{0}^{\infty} x_{m} f_{X, F L I P}\left(x_{m}\right) d x_{m}=\frac{\kappa \sigma_{F L I P}}{\sqrt{2 \pi}}$

and

$P_{e, F L I P}=E\left(\left(x_{m}^{+}\right)^{2}\right)=E\left(\left(x_{m}^{-}\right)^{2}\right)=\kappa \int_{0}^{\infty} x_{m}^{2} f_{X, F L I P}\left(x_{m}\right) x_{m}=\frac{\kappa \sigma_{F L I P}^{2}}{2}$

where $\kappa$ is a control parameter for the signal $x_{m}$ depending on the modulation order.

At the receiving end, the FLIP scheme signal is recovered by subtracting the negative signal component from the positive component [18]. The eventual optical and electrical SNR of the FLIP scheme are given respectively as:

$S N R_{O, F L I P}=\frac{E\left(x_{m}^{+}\right)}{b_{F L I P} N_{o}}=\frac{E\left(x_{m}^{-}\right)}{b_{F L I P} N_{o}}$

and

$S N R_{e, F L I P}=\frac{E\left(\left(x_{m}^{+}\right)^{2}\right)}{b_{F L I P} N_{O}}=\frac{E\left(\left(x_{m}^{-}\right)^{2}\right)}{b_{F L I P} N_{o}}$

where, $b_{F L I P}$ is the throughput of the FLIP scheme while all other parameters assume their usual meaning

The relationship between average BER and the SNR for FLIP scheme follows directly from the derivations in Equation (36) to Equation (39) and are given as follows:

$$
\begin{aligned}
& S N R_{o, F L I P}=\frac{E\left(x_{m}^{+}\right)}{\frac{\sum_{l=1}^{N / 2-1} b_{l, F L I P} B E R_{l}}{B E R \text { ave }} N_{o}} \\
& S N R_{O, F L I P}=\frac{B E R_{\text {ave }} \cdot E\left(x_{m}^{+}\right)}{N_{o} \sum_{l=1}^{N / 2-1} b_{l, F L I P} B E R_{l}} \\
& S N R_{e, F L I P}=\frac{E\left(\left(x_{m}^{+}\right)^{2}\right)}{\frac{\sum_{l=1}^{N / 2-1} b_{l, F L I P} B E R_{l}}{B E R_{a v e}} N_{o}} \\
& S N R_{e, F L I P}=\frac{B E R_{\text {ave }} \cdot E\left(\left(x_{m}^{+}\right)^{2}\right)}{N_{o} \sum_{l=1}^{N / 2-1} b_{l, F L I P} B E R_{l}} .
\end{aligned}
$$

\section{RESULTS}

In estimating the performance of the VLC-based multicarrier schemes, their performance relative to selected quadrature amplitude modulation (QAM) constellations for broadband application is adopted. The validated constellations are 4QAM, 16QAM, 64QAM and 256QAM respectively [21] which were evaluated in relation to the multicarrier schemes for a threshold bit error rate of $10^{-4}$ and a total number of $\mathrm{N}=1024$ subcarriers. To further improve on the modulation schemes' BER response, Artificial Neural network (ANN) was adopted as the machine learning algorithm for channel estimation shown in Figure 2 to adaptively improve the BER response relative to increasing SNR and the channel condition considering a typical white LED driving voltage of 3.5V [22-23]. ANN is a machine learning algorithm based on the 
ideology of human neurons consisting of the input, hidden and output layers. It resolves optimization problems by training a given dataset to enable the correlation between the given input and an expected output. Levenberg Marquardt is adopted during the dataset training since it combines the stability of error back propagation and the speed of Gauss-newton's algorithms. The Levenberg Marquardt algorithm update rule is given by:

$$
W_{k+1}=w_{k}-\left(J_{K}^{T} J_{k}+\mu I\right)^{-1} J_{k} e_{k} .
$$

where; $\mu=$ Combination Coefficient (Positive Value), $I=$ Identity Matrix, $w=$ weight vector, $e=$ training error and $J=$ Jacobian matrix.

Figure 2 (a-e) is a single algorithmic flowchart that has been sectionalized using the continuity symbol (pentagon shape) to depict their interrelationship. Figure $2 \mathrm{a}$ and Figure $2 \mathrm{~b}$ depicts the transmission side of the VLC link. The transmitted serial bit sequence $x_{t}$ is first symbol sorted and transmitted via narrower bands ranging from 0 to $n$ channels. The parallelized signals then take the format $x_{0}, x_{1}, \ldots, x_{n}$ and are mapped to the digital format $X_{0}, X_{1}, \ldots, X_{N-1}$ depending on the selected modulation scheme. The modulated output from each sub-band is then processed to suppress the imaginary component by imposing Hermitian symmetry since the real component is exclusively required for intensity modulation after performing the IFFT operation to convert the signal into discrete time domain samples shown in Figure 2a. It is to be noted that the pentagon symbol shown at the tail end of Figure $2 a$, beginning and end of Figure $2 b$ to Figure $2 d$ and the beginning of Figure $2 \mathrm{e}$ represents continuity of the algorithmic flowchart. Figure $2 \mathrm{~b}$ follows directly from Figure 2a such that cyclic prefix is added to the discrete time domain samples to enforce resistance to inter-symbol interference before serializing the transmitted parallel symbols. The digital samples are then converted to analog format capable of driving the transmitter. It is to be noted that broken lines are used to interface the Digital/Analog Converter and the scheme used to achieve the real and unipolar signal largely because of the ingenuity required in the proposition of novel techniques required to achieve real and unipolar signals which could be DCO, ACO, and FLIP. The processed real valued and unipolar signal is then used to drive the LED transmitter for intensity modulation whose performance is measured by passing its output through a decision feedback to ascertain error free transmission. The modulated intensity shown in the algorithmic flowchart of Figure $2 b$ is directly detected by the direct detection scheme usually a photodetector shown in Figure $2 \mathrm{c}$ and fed to the input of an analog to digital converter before de-cyclicprefixing. 


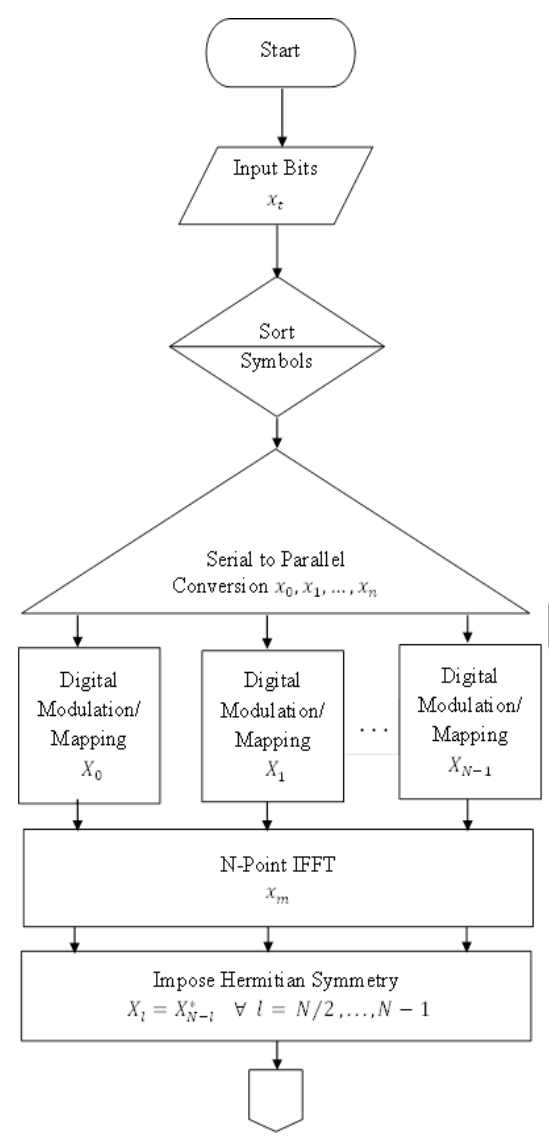

(a)

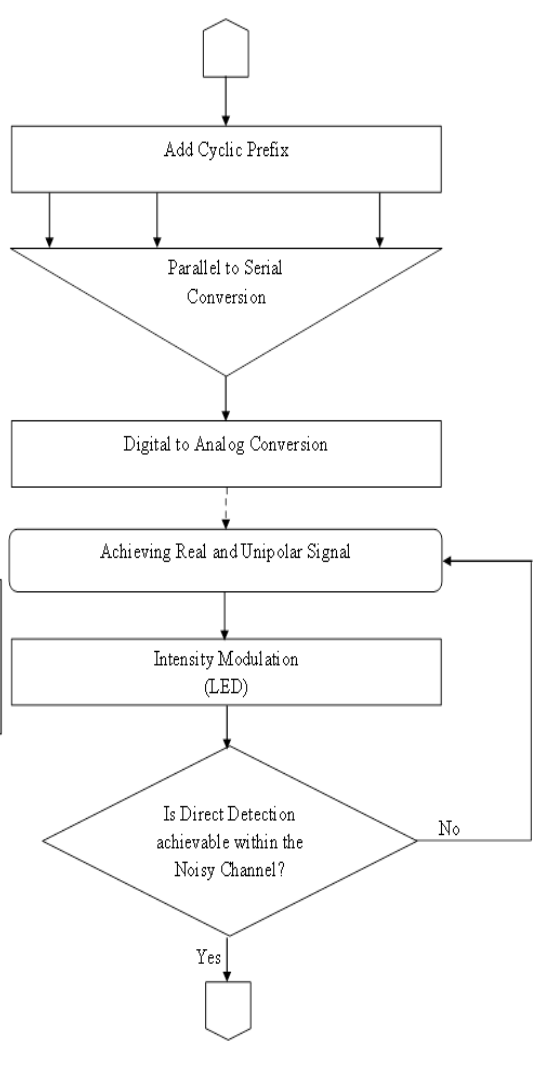

(b)

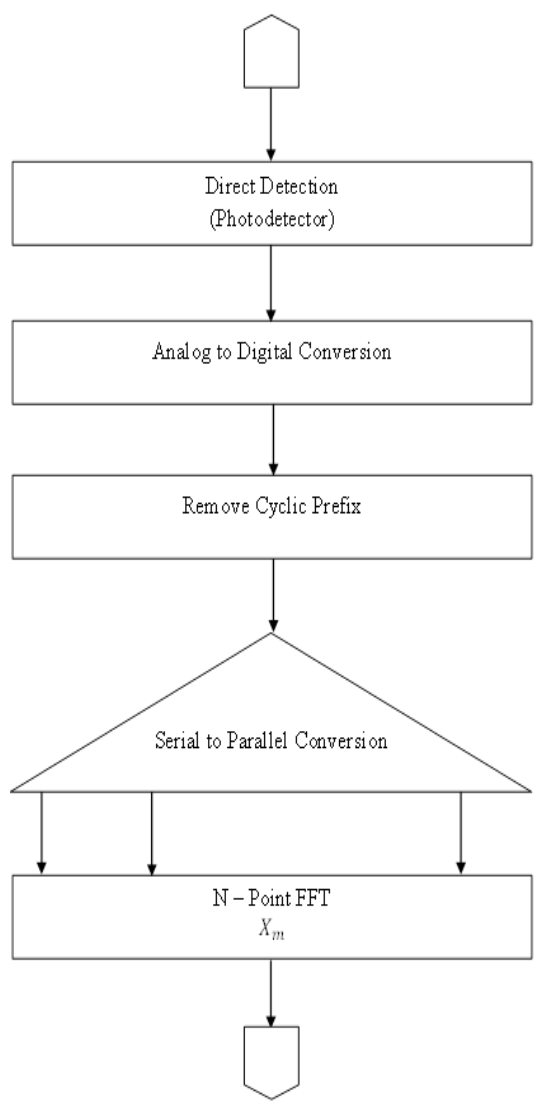

(c)

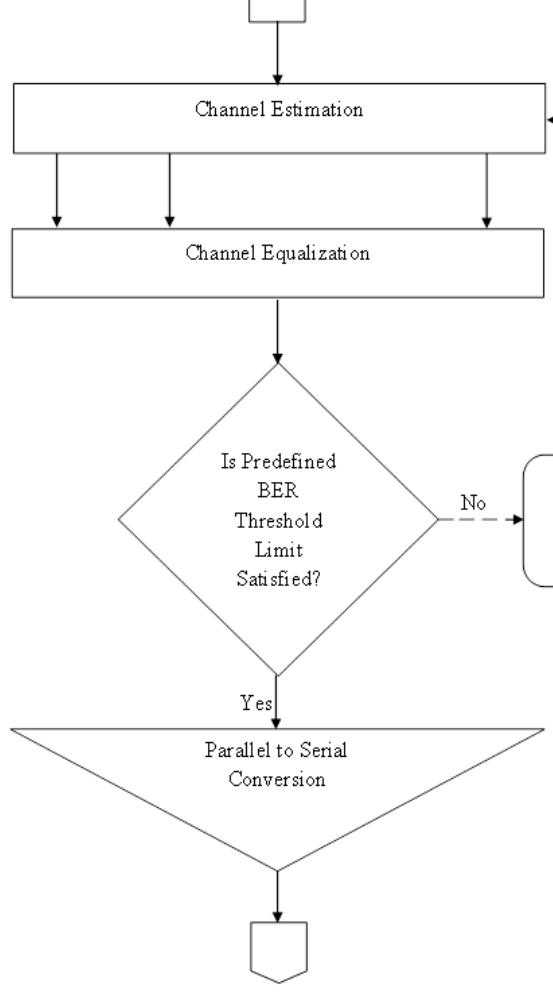

(d)

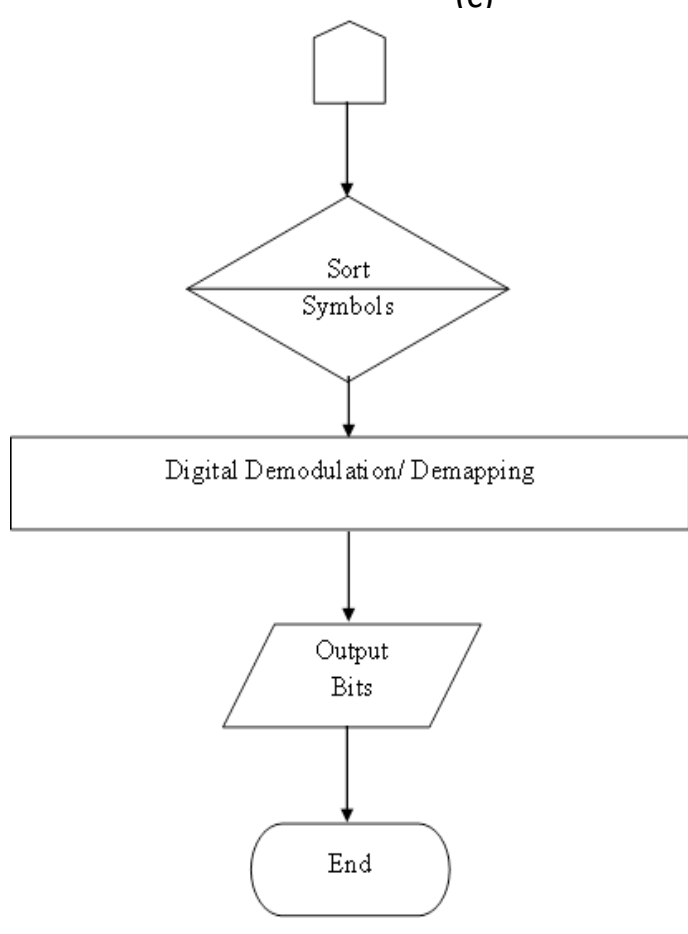

(e)

Figure $2(\boldsymbol{a}-\boldsymbol{e})$. The Machine Learning-based Multicarrier Modulation Algorithmic Flowchart 
The serial signal is once again converted to parallel narrow sub-band signals before transforming the time domain signal into its frequency domain equivalent. The FFT output is then processed by performing channel estimation and equalization to ascertain its BER performance which is critical for error free transmission. Again, a channel optimization scheme is implemented using the machine learning (ANN) algorithm for optimal channel estimation connected to the channel estimation and the decision feedback schemes using the broken lines. This machine learning algorithm is required to improve the overall performance of the VLC link by adaptively minimizing the bit error rate. In the ANN channel estimation module, the received signal component, being the combination of the transmitted signal, channel noise and gain is further trained by the ANN algorithm to enforce correlation between the distorted received signal and the transmitted signal. The optimized signal is then converted to serial format and then symbol sorted, demapped and demodulated for optimal reception as depicted in Figure 2e.

The machine learning training parameters used in this work are shown in Table 1:

Table 1. The Training Parameters of the Machine Learning Algorithm

\begin{tabular}{|l|l|}
\hline Design Architecture & 1 input, 100 hidden layers, 1 output layer and 1 output \\
\hline Training Algorithm & Levenberg-Marquardt (trainlm) \\
\hline Transfer Function & Tansig/purelin \\
\hline Data Division & Random (dividerand) \\
\hline Maximum Training Epoch & 200 \\
\hline Performance Function & MSE \\
\hline Performance Goal & $10^{-5}$ \\
\hline Total No of Hidden Neutrons & 100 \\
\hline
\end{tabular}

The statistical tools used for performance analysis in this work are the root mean squared error (RMSE) and mean absolute percentage error (MAPE) being the relative and absolute error metrics and the regression R-value.

$$
\begin{aligned}
& \text { RMSE }=\sqrt{\frac{\sum_{j=1}^{m}\left(\hat{x}_{k}-x_{k}\right)^{2}}{m} .} \\
& \text { MAPE }=\sum_{k=1}^{m} \frac{\left|\frac{x_{k}-x_{k}}{x_{k}}\right|}{m} \times 100 \% .
\end{aligned}
$$

where $k$ is the sample period, $\hat{x}_{k}$ is the existing or present value, $x_{k}$ is the new or optimized value, $m$ is the duration of the samples.

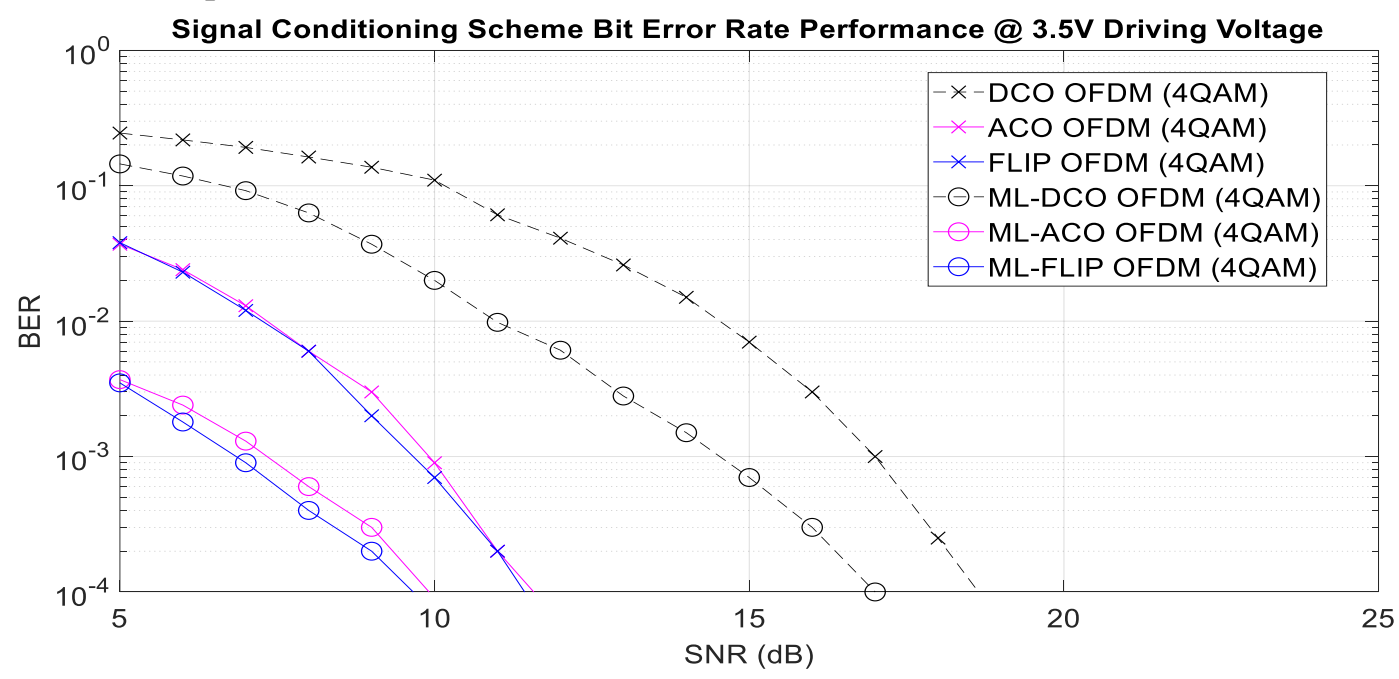

Figure 3. Performance Comparison of BER Response of the Conventional and Machine Learning-based Multicarrier Schemes using $4 Q A M$ 


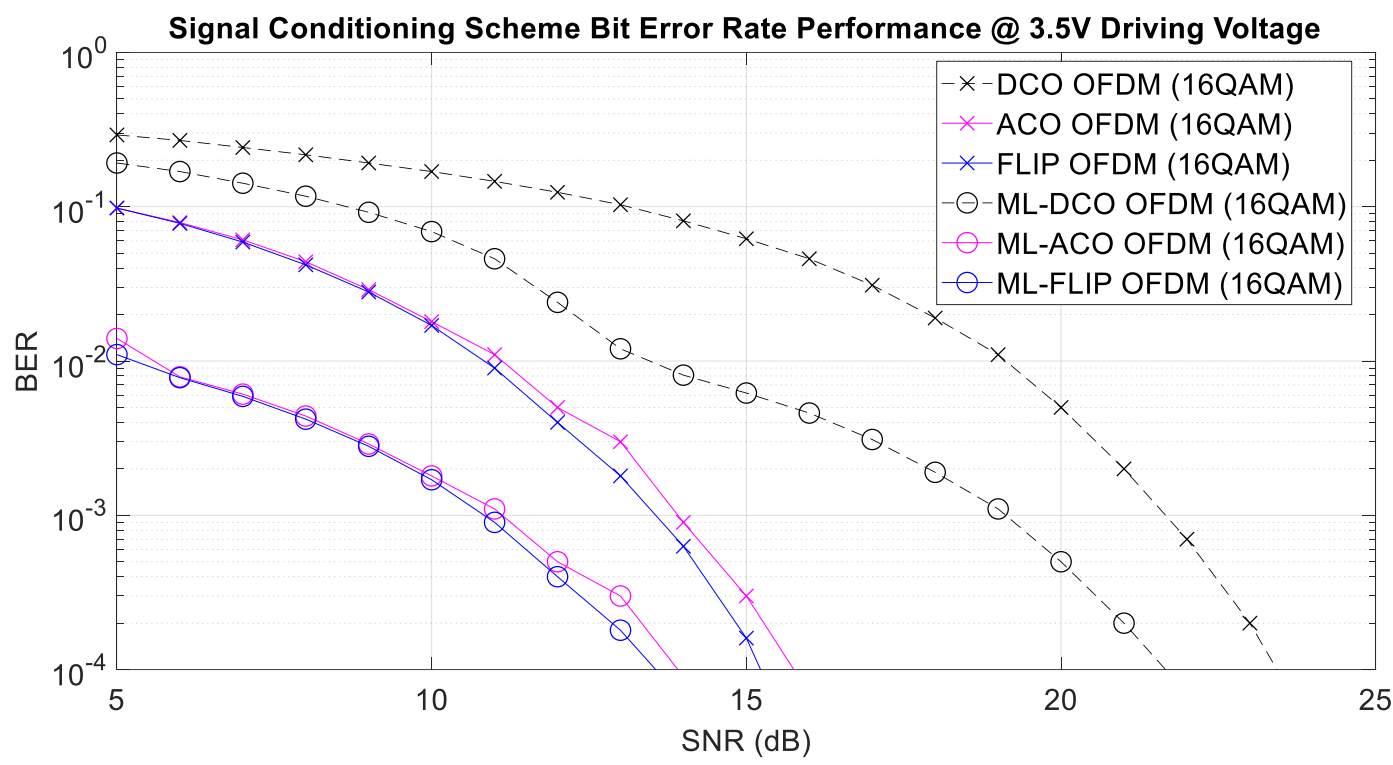

Figure 4. Performance Comparison of BER Response of the Conventional and Machine Learning-based Multicarrier Schemes using 16QAM

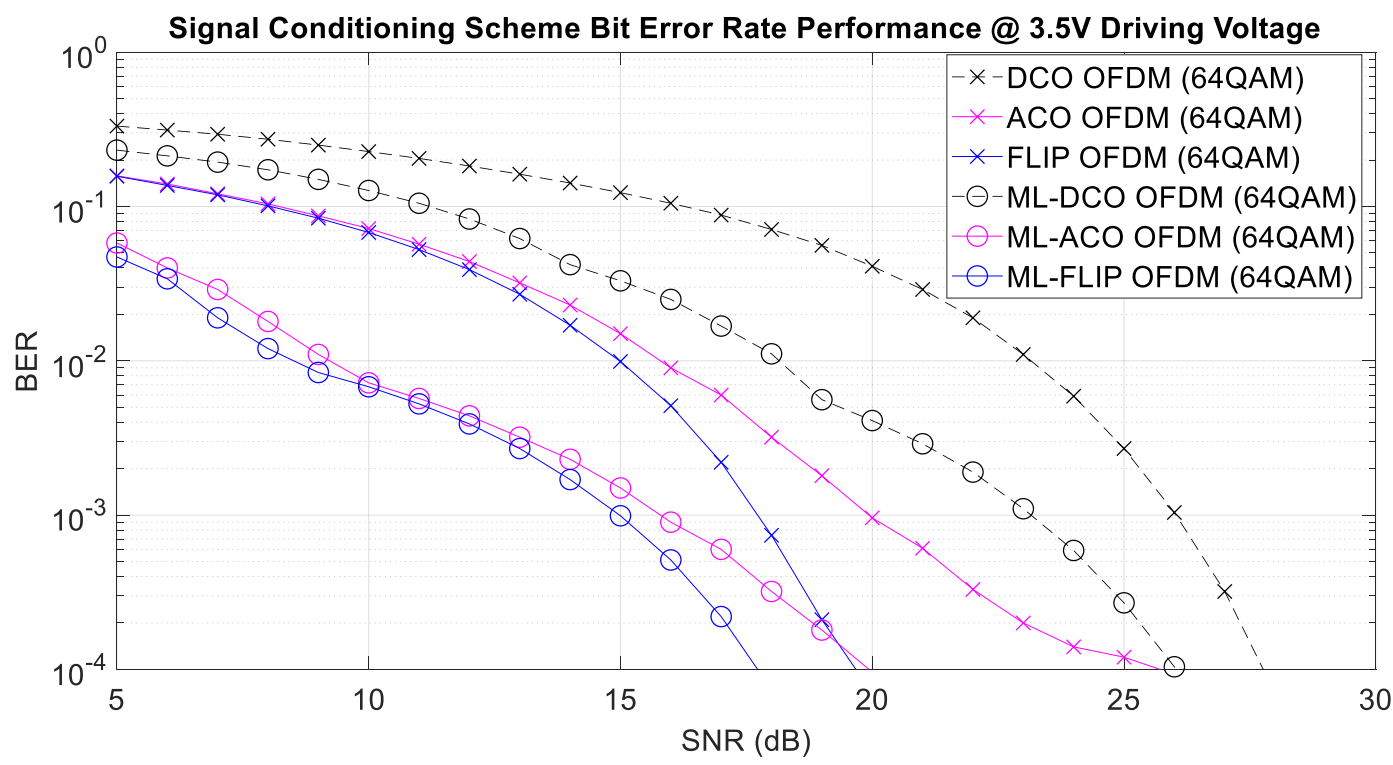

Figure 5. Performance Comparison of BER Response of the Conventional and Machine Learning-based Multicarrier Schemes using 64QAM 


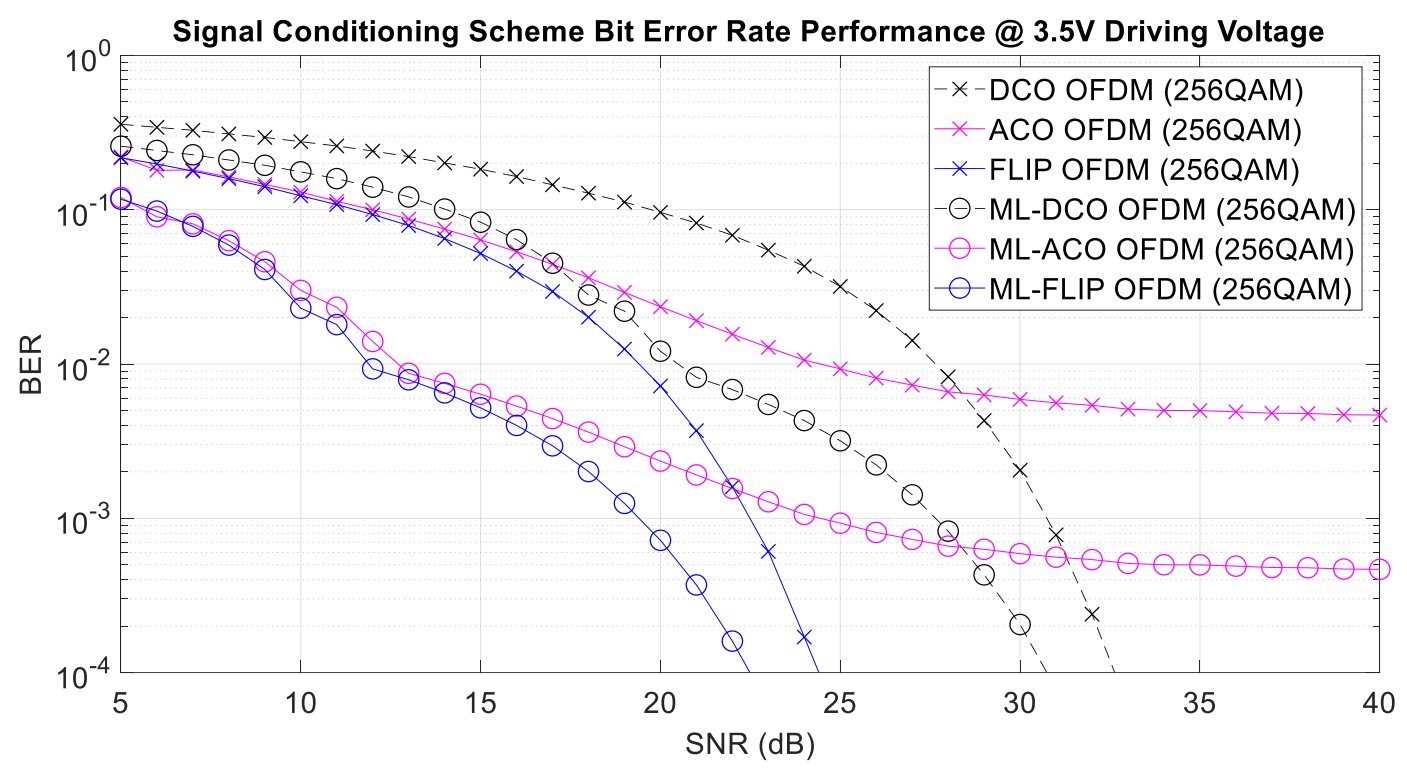

Figure 6. Performance Comparison of BER Response of the Conventional and Machine Learning-based Multicarrier Schemes using 256QAM

The graphs of Figure 3 to Figure 6 depict the BER response of the conventional and machine learning based multicarrier schemes for VLC. Appreciable improvement of the BER response is seen when the channel estimation is conducted using the machine learning algorithm. In general, an average of $1.69 \mathrm{~dB}$ improvement was observed between with and without machine learning for the optical OFDM schemes.

Without the machine learning algorithm, using the constellation size of 4QAM, ACO was seen to outperform DCO with about $2.81 \mathrm{~dB}$ improvement while FLIP outperformed ACO with about $0.45 \mathrm{~dB}$. Similarly, for the constellation size of 256QAM, ACO showed an appreciable improvement over DCO up to $1.73 \mathrm{~dB}$ with FLIP outperforming ACO with about $0.41 \mathrm{~dB}$. Table 2 further depicts the improvement without the machine learning algorithm for 16QAM and 64QAM respectively.

With the machine learning algorithm, the performances of the optical OFDM schemes were compared as shown in Table 3. The machine learning-based schemes were seen to outperform the conventional schemes with an appreciable margin. For the constellation size of 4QAM, ML-DCO, ML-ACO and ML-FLIP showed an appreciable improvement over DCO, ACO and FLIP with about $2.04 \mathrm{~dB}, 2.23 \mathrm{~dB}$ and $2.72 \mathrm{~dB}$ respectively. The corresponding improvements for 16QAM, 64QAM and 256QAM are shown in Table 3 accordingly.

The performance comparison of the machine learning-based schemes using 4QAM constellation showed a notable improvement of ML-ACO over ML-DCO and ML-FLIP over ML-ACO with about $2.01 \mathrm{~dB}$ and $0.61 \mathrm{~dB}$ respectively. Table 4 depicts the corresponding improvements of ML-ACO over ML-DCO and ML-FLIP over ML-ACO for the constellation sizes of 4QAM, 16QAM, 64QAM and 256QAM respectively.

Table 2. Optical Modulation Comparison without Machine Learning

\begin{tabular}{|l|l|l|l|l|}
\hline & 4QAM $(\mathrm{dB})$ & 16QAM $(\mathrm{dB})$ & 64QAM $(\mathrm{dB})$ & 256QAM $(\mathrm{dB})$ \\
\hline ACO vs. DCO & 2.81 & 2.55 & 0.83 & 1.73 \\
\hline FLIP vs. ACO & 0.45 & 0.72 & 0.86 & 0.41 \\
\hline
\end{tabular}


Table 3. Optical Modulation Comparison with and without Machine Learning

\begin{tabular}{|l|l|l|l|l|}
\hline & 4QAM $(\mathrm{dB})$ & $16 \mathrm{QAM}(\mathrm{dB})$ & $64 \mathrm{QAM}(\mathrm{dB})$ & $256 \mathrm{QAM}(\mathrm{dB})$ \\
\hline $\begin{array}{l}\text { ML-DCO vs. } \\
\text { DCO }\end{array}$ & 2.04 & 2.01 & 1.96 & 1.96 \\
\hline $\begin{array}{l}\text { ML-ACO vs. } \\
\text { ACO }\end{array}$ & 2.23 & 2.25 & 2.46 & 2.36 \\
\hline $\begin{array}{l}\text { ML-FLIP vs. } \\
\text { FLIP }\end{array}$ & 2.72 & 2.25 & 2.20 & 2.15 \\
\hline
\end{tabular}

Table 4. Optical Modulation Comparison with Machine Learning

\begin{tabular}{|l|l|l|l|l|}
\hline & 4QAM $(\mathrm{dB})$ & $16 \mathrm{QAM}(\mathrm{dB})$ & $64 \mathrm{QAM}(\mathrm{dB})$ & $256 \mathrm{QAM}(\mathrm{dB})$ \\
\hline ML-ACO vs. ML-DCO & 2.01 & 2.46 & 0.45 & 1.99 \\
\hline ML-FLIP vs. ML-ACO & 0.61 & 0.76 & 1.04 & 1.17 \\
\hline
\end{tabular}

For the constellation size of 4QAM, the relative and absolute errors were seen to be $0.06922,0.00513$ and 0.00664 RMSEs and $1.415 \times 10^{50}, 39516.46$ and 1432.46 MAPEs for ML-DCO, ML-ACO and ML-FLIP respectively with an R-score of 0.999999 . With 16QAM constellation, $0.07521,0.00991$ and 0.01196 RMSEs; $2.15 \times 10^{64}, 3402944.28$ and 9423.52 MAPEs for ML-DCO, ML-ACO and ML-FLIP respectively and $0.999992 \mathrm{R}$-score was observed. As higher constellation sizes were used, 0.08062, 0.01352 and 0.01561 RMSEs; $4.01 \times 10^{57}, 4.11 \times 10^{24}$ and 6415.20 MAPEs for ML-DCO, ML-ACO and ML-FLIP respectively and 0.999999 R-score was recorded for 64QAM and 0.09034, 0.02668 and 0.02747 RMSEs; $4.13 \times 10^{60}, 4.53 \times 10^{23}$ and 9197.74 MAPEs for ML-DCO, ML-ACO and ML-FLIP respectively and with 0.98987 R-score for 256QAM. Tables 5 and 6 depict the summary of the relative and absolute errors for the conventional and machine learning-based schemes.

The machine learning-based schemes were seen to show performance superiority relative to the conventional schemes with remarkable reduction in both relative and absolute errors. With such improvement in the BER response relative to increasing SNR values, the schemes invariably will outperform the conventional schemes with higher throughput as fewer bits will be transmitted in error.

Table 5. RMSE Performance measure of the multicarrier schemes

\begin{tabular}{|l|l|l|l|l|}
\hline \multicolumn{5}{|c|}{ RMSE } \\
\hline & 4QAM & 16QAM & $64 \mathrm{QAM}$ & 256QAM \\
\hline DCO & 0.11997 & 0.13221 & 0.14361 & 0.15890 \\
\hline ML-DCO & 0.06922 & 0.07521 & 0.08062 & 0.09034 \\
\hline ACO & 0.02475 & 0.03184 & 0.04697 & 0.07149 \\
\hline ML-ACO & 0.00513 & 0.00991 & 0.01352 & 0.02668 \\
\hline FLIP & 0.02432 & 0.03168 & 0.04594 & 0.06918 \\
\hline ML-FLIP & 0.00664 & 0.01196 & 0.01561 & 0.02747 \\
\hline
\end{tabular}

Table 6. MAPE Performance measure of the multicarrier schemes

\begin{tabular}{|l|l|l|l|l|}
\hline \multicolumn{5}{|c|}{ MAPE } \\
\hline & $4 \mathrm{QAM}$ & $16 \mathrm{QAM}$ & $64 \mathrm{QAM}$ & $256 \mathrm{QAM}$ \\
\hline DCO & $2.10687 \mathrm{E}+65$ & $2.99112 \mathrm{E}+65$ & $3.71759 \mathrm{E}+58$ & $4.10066 \mathrm{E}+61$ \\
\hline ML-DCO & $1.41518 \mathrm{E}+50$ & $2.1536 \mathrm{E}+64$ & $4.01499 \mathrm{E}+57$ & $4.13346 \mathrm{E}+60$ \\
\hline ACO & 434959.8513 & 31435460.06 & $4.1057 \mathrm{E}+25$ & $4.5317 \mathrm{E}+24$ \\
\hline ML-ACO & 39516.45752 & 3402944.275 & $4.1123 \mathrm{E}+24$ & $4.535 \mathrm{E}+23$ \\
\hline FLIP & 15081.32384 & 86388.83977 & 63667.80671 & 1142285658 \\
\hline ML-FLIP & 1432.456583 & 9423.517892 & 6415.200862 & 9197.736619 \\
\hline
\end{tabular}




\section{CONCLUSION}

It was observed that the BER response improved as a result of the adaptive learning process of the machine learning algorithm for all multicarrier modulation schemes. The adoption of the proposed machine learningbased multicarrier modulation (MLMM) scheme can be adopted depending on the spectral and power efficiency requirement as it outperformed its conventional counterpart.

\section{ACKNOWLEDGEMENT}

The authors gratefully acknowledge the sponsorship of this research by the Petroleum Technology Development Fund (PTDF) under the grant award number P4567720076521527.

\section{CONFLICTS OF INTEREST}

No conflict of interest was declared by the authors.

\section{REFERENCES}

[1] Feng, S., Zhang, R., Xu, W., Hanzo, L., "Multiple Access Design for Ultra-Dense VLC Networks: Orthogonal vs Non-Orthogonal ", IEEE Transactions on Communications, 67(3): 2218 - 2231, (2019).

[2] Agboje, O. E., Idowu-Bismark, O. B., Ibhaze, A. E., "Comparative Analysis of Fast Fourier Transform and Discrete Wavelet Transform Based MIMO-OFDM", International Journal on Communications Antenna and Propagation (I.Re.C.A.P.), 7(2): 168 - 175, (2017).

[3] Ndujiuba C. U., Ibhaze, A. E., "Dynamic Differential Modulation of Sub-Carriers in OFDM", Journal of Wireless Networking and Communications, 6(1): 21-28, (2016).

[4] Ibhaze, A. E., Orukpe, P. E., Edeko, F. O., "Li-Fi Prospect in Internet of Things Network", in: J. Kacprzyk (Ed.), FICC2020, Advances in Intelligent Systems and Computing. Cham, Switzerland: Springer Nature Switzerland AG, 1129: 272-280, (2020).

[5] Huang, X., Yang, F., Zhang, H., Ye, J., Song, J., "Subcarrier and Power Allocations for Dimmable Ehanced ADO-OFDM with Iterative Interference Cancellation", IEEE Access, 7: 28422 - 28435, (2019).

[6] Shannon, C. E., "A Mathematical Theory of Communication", The Bell System Technical Journal, 27(3, 4): 379-423, 623-656, (1948).

[7] Ibhaze, A. E., Orukpe, P. E. and Edeko, F. O., "High-Capacity Data Rate System: Review of Visible Light Communications Technology", Journal of Electronic Science and Technology. DOI: https://doi.org/10.1016/j.jnlest.2020.100055, (2020).

[8] Cover T. M. and Thomas, J. A., Elements of Information Theory, 2nd ed. Hoboken, New Jersey: John Wiley \& Sons, Inc., (2006).

[9] Tan, J., Wang, Z., Wang, Q., Dai, L., "BICM-ID scheme for clipped DCO-OFDM in visible light communications", Optics Express, 24(5): 4573 - 4581, (2016).

[10] Nee, R., Awater, G., Morikura, M., Takanashi, H., Webster, M., Halford, K. W., "New High-Rate Wireless LAN Standards", IEEE Communications Magazine, 37(12): 82 - 88, (1999). 
[11] Hu, W. W., "PAPR Reduction in DCO-OFDM Visible Light Communication Systems Using Optimized Odd and Even Sequences Combination", IEEE Photonics Journal, 11(1): 790115, (2019).

[12] Proakis, J. G., Digital Communications, 4th ed, McGrawHill, (2000).

[13] Kahn, J. M., Barry, J. R., "Wireless Infrared Communications", Proceedings of the IEEE, 85(2): 65 - 298, (1997).

[14] Armstrong, J. "OFDM for optical communications", IEEE Journal of Light Wave Technology, 27(3): 189 - 204, (2009).

[15] Devasmitha Dissanayake, S., Armstrong, J., "Comparison of ACO-OFDM, DCO-OFDM and ADOOFDM in IM/DD Systems", IEEE Journal of Lightwave Technology, 31(7): 1063 - 1072, (2013).

[16] Chen, L., Krongold, B., Evans, J. "Performance Analysis for Optical OFDM Transmission in ShortRange IM/DD Systems", IEEE Journal of Lightwave Technology, 30(7): 974 - 983, (2012).

[17] Chung, S. T., Goldsmith, A. J., "Degrees of Freedom in Adaptive Modulation: A Unified View", IEEE Transactions on Communications, 49(9): 1561 - 1571, (2001).

[18] Fernando, N., Hong, Y., Viterbo, E., "Flip-OFDM for Optical Wireless Communications", in IEEE Information Theory Workshop, Paraty, Brazil, 5 - 9, (2011).

[19] Tsonev, D., Haas, H., "Avoiding spectral efficiency loss in unipolar OFDM for optical wireless communication", in IEEE International Conference on Communications (ICC), Sydney, NSW, Australia, 3336 - 3341, (2014).

[20] Wu, L., Zhang, Z., Dang, J., Wang, J., Liu, H., "Polarity Information Coded Flip-OFDM for Intensity Modulation Systems", IEEE Communications Letters, 20(8): 1089 - 7798, (2016).

[21] Castel, T., Lemey, S., Agneessens, S., Torre, P. V., Rogier, H., Oestges, C., "Adaptive subcarrier modulation for indoor public safety body-to-body networks", in IEEE 10th European Conference on Antennas and Propagation (EuCAP), Davos, Switzerland, 1-5, (2016).

[22] Ibhaze, A. E., Orukpe P. E., Edeko, F. O., "Visible Light Channel Modeling for High-data Transmission in the Oil and Gas Industry", Journal of Science and Technology, 12(2): 46-54, (2020).

[23] Nachbaur, O., "White LED Power Supply Design Techniques", Texas Instruments Incorporated, Dallas, Texas, (2003). 